\title{
Spatial and temporal fading correlation of uniform linear antenna array in three- dimensional signal scattering
}

\begin{abstract}
In practice, diffraction and scattering from oblique surfaces create waves that travel in threedimensional (3-D) space and arrive at the receiver with both azimuth and elevation angles. In this paper, 3-D signal scattering is considered. Generalized analytical expressions for the spatial and temporal signal fading correlations are derived, and numerically evaluated, for a uniform linear array (ULA) in terms of the antenna elements spacing and statistical distributions of the angles of arrival (AOAs). The results show that the effect of the elevation angle distribution on the spatial correlation values in case of vertically separated antenna elements is much more significant than that on horizontally separated antenna elements.
\end{abstract}

Keyword: Correlation functions; Fading channels; Linear antenna arrays 